\title{
A partial hypopituitarism case that resolved following bariatric surgery
}

Mitan A, Irshad F, Bukhari S, Nyunt A

Diabetes and Endocrinology Department, Glan Clwyd Hospital, LL18 5UJ

\section{Background}

The use of opioids in non-cancer patients has increased dramatically over the past few years. The most common endocrine dysfunction with opioid use is hypogonadism, but it has been reported in a small number of cases that adrenal insufficiency and adult growth hormone deficiency can also occur.

A 39 year old male presented with general fatigue, loss of libido and sweating for about six months in 2008. He suffered from severe back pain, neck pain and was on morphine sulphate tablet $30 \mathrm{mg}$ BD. The past medical history included T2DM and obesity. In clinic he was overweight (BMI 42) with sparse body hair. His blood tests showed testosterone 5.7 nmol/L, FSH 1.9 U/L, LH 0.8 U/L, Prolactin 514, TSH 2.9mU/L, FT4 $12 \mathrm{pmol} / \mathrm{L}$ and 9 am Cortisol 63nmol/L. MRI pituitary was normal. His partial hypopituitarism was likely due to chronic opioid use. He was commenced on hydrocortisone and testosterone, followed by growth hormone replacement after having dynamic pituitary function tests. In December 2015 he underwent bariatric surgery and lost $36 \mathrm{~kg}$ weight in total. In 2016 his BMI was 33.6; he was no longer on morphine tablets or any treatment for diabetes (HbA1c $42 \mathrm{mmol} / \mathrm{mol}$ ). In 2017, repeat dynamic tests showed his peak GH and cortisol levels were satisfactory. At present, he remains only on testosterone replacement.

This is one of very few cases demonstrating the effect of opioids on $\mathrm{GH}$ levels, other than causing hypogonadism. More research is needed to determine which opioids are more likely to cause endocrine dysfunction and which patients need to be screened and treated. 